\title{
Storage capacity of holographic associative memories
}

\author{
John Hong and Demetri Psaltis
}

Department of Electrical Engineering, California Institute of Technology, 116-81, Pasadena, California 91125

Received June 13, 1986; accepted September 4, 1986

\begin{abstract}
The storage capacity of holographic associative memories is estimated. An argument based on the available degrees of freedom shows that the number of patterns that can be stored is limited by the space-bandwidth product of the hologram divided by the number of pixels in each pattern. A statistical calculation shows that if we attempt to store associations by multiply exposing the hologram, the cross talk among the stored items severely degrades the output fidelity. This confirms the storage capacity predicted by the degrees-of-freedom argument.
\end{abstract}

An associative memory internally stores a set of distinct output signal vectors $\mathrm{g}_{m}, i=1,2, \ldots, M$ in a oneto-one association with a second set of stimulus signals $\mathbf{f}_{m}$ in such a way as to make selective recall possible. That is, a signal $\mathbf{g}_{k}$ is recalled by presenting its associated stimulus, $\mathbf{f}_{k}$, as the input. Such a memory is sometimes described as being heteroassociative, of which autoassociative schemes in which the stimulus and stored signals are the same form a special case.

The analogy between associative memories and holography was first pointed out by Van Heerden ${ }^{1}$ and also by Gabor. ${ }^{2}$ A hologram records the interference pattern between two waves, one of which is normally referred to as the reference wave and the other as the signal. When the hologram is illuminated with the reference wave, the diffraction due to the stored interference pattern results in the reconstruction of the signal.

Whereas Gabor discussed the holographic technique for only one association, recent publications have discussed the possibility of storing many associations on a single hologram..$^{3-10}$ It is therefore important to quantify the storage capacity of holographic associative memories, defined to be the maximum number of associations that can be stored and recalled with high fidelity. In this Letter we present a discussion based on the degrees-of-freedom argument forwarded by Abu-Mostafa, and Psaltis ${ }^{12}$ demonstrates the fundamental importance of the space-bandwidth product (SBP) of the hologram in determining the storage capacity. Specifically, we show that the total number of samples or bits that can be stored is equal to the SBP. This implies that the storage capacity of holographic associative memories is limited to one association if we wish to store images each with a SBP equal to that of the hologram. This result is then confirmed by deriving an expression of the signal-tonoise ratio (SNR) that is obtained when the hologram is reconstructed.

In a conventional hologram, two patterns are assoc1ated with each other by recording their mutual interference. More generally, a hologram can be thought of as an optical transparency whose complex transmittance can be controlled arbitrarily. Information is stored in the hologram such that when it is placed in the appropriate optical system and the system is illu- minated by one of the specified inputs, the correct, associated output is produced. A model that is sufficiently general to encompass all the specific holographic memories is shown in Fig. 1. We will use this model to derive bounds on the capacity that are generally applicable without requiring further knowledge of the implementation details. The information content of a hologram is characterized primarily by its SBP, and therefore the system spatial coordinate is quantized by assigning $N_{2}=\mathrm{SBP}$ discrete pixels to the hologram. Similarly, the input and output planes are composed of $N_{1}$ and $N_{3}$ pixels, respectively. The input, output, and hologram planes have been identified in Fig. 1. To be general, arbitrary, but fixed, linear transformations $Q(j, i)$ and $R(k, j), j=1,2, \ldots, N_{2}$; $i=1,2, \ldots, N_{1} ; k=1,2, \ldots, N_{3}$ relate the input and output planes to the hologram plane.

This system pairwise stores $N_{1}$-element vectors from a set of $M$ inputs $f_{m}(i)$, where $m$ is the memory index, with $N_{3}$-element vectors from the set of $M$ outputs $g_{m}(k)$, such that the reconstruction of the vector $g_{m_{0}}(k)$ is obtained when its associated vector $f_{m_{0}}(i)$ is present at the input plane. Given the freedom to write the hologram in any way we choose, the reconstruction process is studied in detail to determine the maximum number of associations, $M$.

In reference to Fig. 1, the input-output relation that must be satisfied for selective recall can be described by the following equations:

$$
\begin{aligned}
g_{m}(k)= & \sum_{j=1}^{N_{2}} \sum_{i=1}^{N_{1}} R(k, j) t_{H}(j) Q(j, i) f_{m}(i) \\
& \quad \text { for } k=1,2, \ldots, N_{2}, \quad m=1,2, \ldots, M,
\end{aligned}
$$

where $t_{H}(j), j=1,2, \ldots, N_{2}$ are the pixel values that describe the hologram's transmittance. Since Eq. (1) is a system of $M N_{3}$ equations in the $N_{2}$ unknowns $t_{H}(j)$, a solution $t_{H}(j)$ is guaranteed only if $M N_{3} \leq N_{2}$. This provides an upper bound for the capacity of the holographic memory: $M \leq N_{2} / N_{3}$, where $M$ is the number of associations and $N_{3}$ is the size of each output. Thus there is a trade-off in the memory capacity, and the fundamental limit is set by the SBP of the hologram. The equality constraints placed by Eqs. (1) may appear to be severe, and one might wonder 


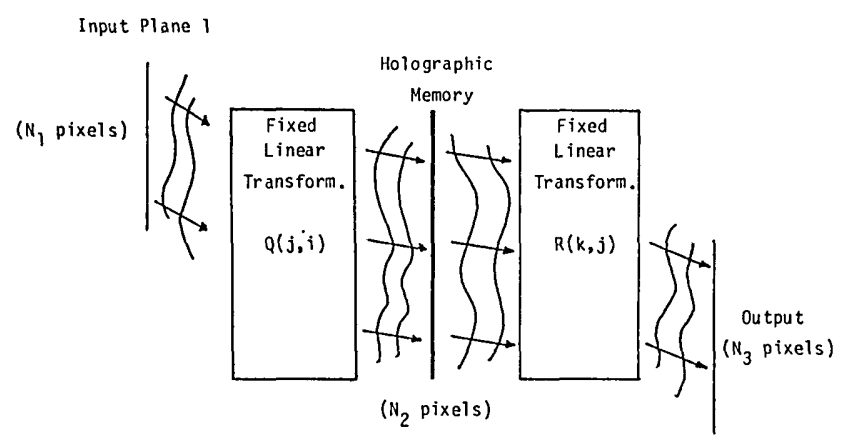

Fig. 1. General holographic model.

whether the storage capacity would increase substantially if we required only approximate reconstruction. For instance, if we require only that the output of the holographic memory have the correct sign rather than the exact analog value, we can use Cover's result for the capacity of linear discriminant functions to conclude that the capacity does not increase at all. We believe that this conclusion is not specific to this particular type of inaccuracy. In what follows, we analyze another approximate method of constructing optical associative memories that also confirms this conclusion.

A heuristic method of assigning values to $t_{H}(j)$ to satisfy the constraints of Eq. (1) approximately is found in optical holography. Conventional holograms are prepared by recording on a photographic plate or any other suitable recording medium the mutual interference pattern between a pair of impinging waves that arise from the diffraction of optical field amplitudes at some defined input planes. This is shown in Fig. 2. For generality, we consider fixed, linear transformations to relate both input planes to the hologram plane. One wave is then reconstructed by illuminating the developed plate with the other. Pairs of data are stored by multiply exposing the hologram, resulting in a holographic-plate transmittance function that is a superposition of the interference patterns of the stored waves. If one particular wave is used as the input for the reconstruction, the output will consist of the wave associated with that input but distorted by a cross-correlation noise term composed of the other stored waves. A good performance criterion is then the SNR, defined as the ratio of the correct output signal amplitude to the standard deviation of the cross-correlation noise term. We now consider this holographic paradigm in which $t_{H}(j)$ is a superposition of products of linearly transformed vectors. In reference to Fig. 2, the transmittance of the hologram is described by

$$
t_{H}(j)=\sum_{m=1}^{M}\left[\sum_{l=1}^{N_{1}} Q^{*}(j, l) f_{m}^{*}(l)\right]\left[\sum_{j=1}^{N_{3}} S(j, n) g_{m}(n)\right],
$$

where we have omitted inconsequential terms such as bias that also appear in the holographic recording process. The response due to a particular input $f_{m_{0}}(i)$ is

$$
\begin{aligned}
& g(k)=\sum_{j=1}^{N_{2}} R(k, j) t_{H}(j) \sum_{i=1}^{N_{1}} Q(j, i) f_{m_{0}}(i), \\
& k=1,2, \ldots, N_{3},
\end{aligned}
$$

which when expanded gives

$$
\begin{aligned}
g(k)= & \sum_{i=1}^{N_{1}} \sum_{l=1}^{N_{1}} \sum_{j=1}^{N_{2}} \sum_{n=1}^{N_{3}} R(k, j) S(j, n) Q^{*}(j, l) Q(j, i) \\
& \times f_{m_{0}}{ }^{*}(l) f_{m_{0}}(i) g_{m_{0}}(n)+\alpha(k), \\
\alpha(k)= & \sum_{m \neq m_{0}}^{M} \sum_{i=1}^{N_{1}} \sum_{l=1}^{N_{1}} \sum_{j=1}^{N_{2}} \sum_{n=1}^{N_{3}} R(k, j) S(j, n) Q^{*}(j, l) Q(j, i) \\
& \times f_{m}{ }^{*}(l) f_{m_{0}}(i) g_{m}(n),
\end{aligned}
$$

where the expression for $g(k)$ has been resolved into a signal term and a cross-correlation noise term, $\alpha(k)$. If we assume that the elements of the vectors $f_{m}(i)$ and $g_{m}(n)$ take the value +1 or -1 with equal probability and that each element is statistically independent from all others, then the expected value of the output is

$$
E[g(k)]=\sum_{n=1}^{N_{3}} \sum_{j=1}^{N_{2}} R(k, j) S(j, n) \sum_{i=1}^{N_{1}}|Q(j, i)|^{2} g_{m_{0}}(n),
$$

where we have used the fact that $E\left[f_{m_{0}}(l) f_{m_{0}}(i)\right]=\delta(l$, $i)$, the Kronecker delta function. If we choose the set of possible transformations $Q(j, i)$ such that the row sums $\Sigma_{i=1}^{N_{1}}|Q(j, i)|^{2}=1$ for all $j$, then Eq. (5) further simplifies to

$$
E[g(k)]=\sum_{n=1}^{N_{3}} \sum_{j=1}^{N_{2}} R(k, j) S(j, n) g_{m_{0}}(n) .
$$

For $E[g(k)]$ to be proportional to the correct recall term, $g_{m_{0}}(k), R(k, j)$, and $S(j, n)$ must satisfy the condition

$$
\sum_{j=1}^{N_{2}} R(k, j) S(j, n)=c \delta(k, n),
$$

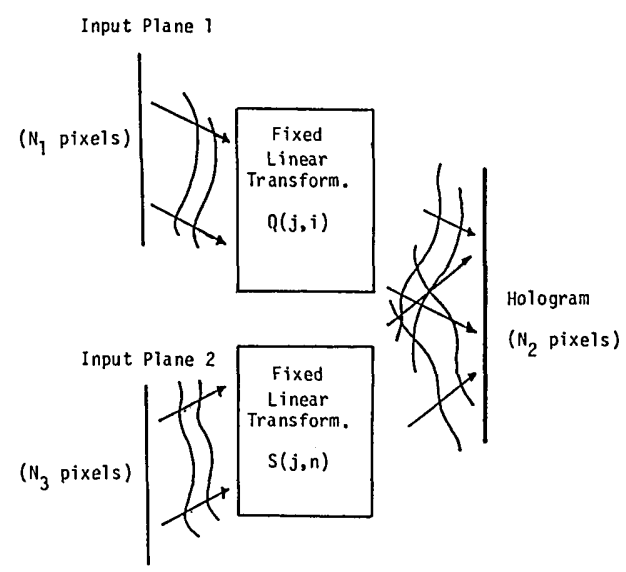

Fig. 2. Holographic recording. 
where $c$ is a constant. The requirement that $\Sigma_{i=1}^{N_{1}} \mid Q(j$, i) $\left.\right|^{2}=1$ that was invoked to derive the above result is not overly restrictive, as it is applicable to most transformations of interest. Specifically, this condition describes transformations that distribute the input energy uniformly over the hologram plane, making efficient use of the space on the hologram. That is, a uniform, incoherent intensity distribution at the input plane 1 of Fig. 1 is mapped to a uniform intensity at the hologram plane. Some examples are Fresnel, Fourier, and diffuse transformations.

Satisfying Eq. (7) guarantees that the expected value of the output will be proportional to the correct stored pattern. In order to obtain an estimate for the variance of the reconstruction, we need to specify the transformations $R(k, j)$ and $S(j, n)$ more precisely. We make the reasonable requirement that the energy dissipated by the transformation $S(j, n)$ not depend on the specific vectors $g_{m}(n)$ being stored. Again, transformations of interest such as Fourier, Fresnel, and diffuse transformations satisfy this condition. Mathematically, this leads to the requirement that $S(j, n)$ have orthogonal columns:

$$
\sum_{j=1}^{N_{2}} S^{*}(j, k) S(j, n) \propto \delta(k, n) .
$$

To satisfy Eq. (7) and relation (8), we require that

$$
S(j, n)=R^{*}(n, j), \quad \sum_{j=1}^{N_{2}} R(k, j) R^{*}(n, j)=c \delta(k, n) .
$$

Having concluded that $E[g(k)]=c q_{m_{0}}(k)$ when Eq. (7) holds, we now calculate the noise variance $\sigma^{2}(k)$ :

$$
\begin{aligned}
\sigma^{2}(k)= & E\left[|\alpha(k)|^{2}\right] \\
= & (M-1) \sum_{i=1}^{N_{1}} \sum_{l=1}^{N_{1}} \sum_{j_{1}=1}^{N_{2}} \sum_{j_{2}=1}^{N_{2}} \sum_{n=1}^{N_{3}} R\left(k, j_{1}\right) R^{*}\left(k, j_{2}\right) \\
& \times R^{*}\left(n, j_{1}\right) R\left(n, j_{2}\right) \\
& \times Q^{*}\left(j_{1}, l\right) Q\left(j_{1}, i\right) Q\left(j_{2}, l\right) Q^{*}\left(j_{2}, i\right) .
\end{aligned}
$$

The index $k$ is kept to show the possible dependence of the variance on the output bit position. To arrive at a more nearly uniform measure of the noise, we calculate the variance averaged over the $N_{3}$ output bits to be

$$
\begin{aligned}
\sigma^{2}= & \left(1 / N_{3}\right) \sum_{k=1}^{N_{3}} \sigma^{2}(k) \\
= & (M-1) / N_{3} \sum_{j_{1}=1}^{N_{2}} \sum_{j_{2}=1}^{N_{2}}\left|\sum_{k=1}^{N_{3}} R\left(k, j_{1}\right) R^{*}\left(k, j_{2}\right)\right|^{2} \\
& \times\left|\sum_{i=1}^{N_{1}} Q\left(j_{1}, i\right) Q^{*}\left(j_{2}, i\right)\right|^{2}
\end{aligned}
$$

A lower bound on the noise variance can be derived by first noting that

$$
\begin{aligned}
& \sigma^{2} \geq\left[(M-1) / N_{3}\right] \\
& \quad \times \sum_{j=1}^{N_{2}}\left[\left.\left.\left.\left|\sum_{k=1}^{N_{3}}\right| R(k, j)\right|^{2}\right|^{2}|| \sum_{i=1}^{N_{1}}|Q(j, i)|^{2}\right|^{2}\right] .
\end{aligned}
$$

But since we have restricted the $Q(i, j)$ 's such that $\Sigma_{i=1}^{N_{1}}|Q(j, i)|^{2}=1$ and also $\Sigma_{k=1}^{N_{3}} \sum_{j=1}^{N_{2}}|(k, j)|^{2}=c N_{3}$ from Eqs. (9), we get

$$
\begin{aligned}
\sigma^{2} & \geq\left.\left.\left[(M-1) / N_{3}\right] \sum_{j=1}^{N_{2}}\left|\sum_{k=1}^{N_{3}}\right| R(k, j)\right|^{2}\right|^{2} \\
& =(M-1) c^{2} N_{3} / N_{2},
\end{aligned}
$$

where the inequality $N_{2} \Sigma_{j=1}^{N_{2}}|a(j)|^{2} \geq\left|\Sigma_{j=1}^{N_{2}} a(j)\right|^{2}$ was used. Using the above lower bound and noting from Eqs. (6) and (7) that the output signal amplitude is $c$, the SNR can be bounded from above as

$$
\begin{aligned}
\mathrm{SNR} & \equiv(\text { signal amplitude }) / \sigma \\
& \leq\left[N_{2} /(M-1) N_{3}\right]^{1 / 2} .
\end{aligned}
$$

The output SNR must be sufficiently large for effective operation of the memory. If we require that SNR $\geq 1$, then we get the capacity bound of

$$
(M-1) \leq N_{2} / N_{3} \text {, }
$$

which is recognized, for $M \gg 1$, to be the bound derived earlier using the degrees-of-freedom arguments.

The authors thank Y. S. Abu-Mostafa, E. Paek, C. H. Park, and F. Mok for many helpful discussions. The research is supported by the Defense Advanced Research Projects Agency and the U.S. Army Research Office. The research of J. Hong was supported in part by a fellowship from Caltech's Program in Advanced Technologies, supported by Aerojet General, General Motors, GTE, and TRW.

\section{References}

1. P. J. van Heerden, Appl. Opt. 2, 387 (1963).

2. D. Gabor, IBM J. Res. Devel. 13, 156 (1969).

3. H. J. Mager, O. Wess, and W. Waidelich, Opt. Commun. 9, 156 (1973).

4. D. Psaltis and N. Farhat, Opt. Lett. 10, 98 (1985).

5. H. Mada, Appl. Opt. 24, 2063 (1985).

6. H. J. Caulfield, Opt. Commun. 55, 80 (1985).

7. B. Soffer, G. J. Dunning, Y. Owechko, and E. Marom, Opt. Lett. 11, 118 (1986).

8. D. Anderson, Opt. Lett. 11, 56 (1986).

9. M. Cohen, Proc. Soc. Photo-Opt. Instrum. Eng. 625, 30 (1986).

10. A. Yariv et al., Proc. Soc. Photo-Opt. Instrum. Eng. 613, 1 (1986).

11. Y.S. Abu-Mostafa and D. Psaltis, presented at the IEEE Computer Society Workshop on Computer Architecture for Pattern Analysis and Image Database Management, Miami Beach, Fla., November 1985. 